\title{
Markus Schwenkreis (Hg.), Compendium Improvisation - Fantasieren nach historischen Quellen des 17. und 18. Jahrhunderts (= Scripta, Bd. 5), Basel: Schwabe 2018
}

Schlagworte/Keywords: Choral; chorale; Fantasie; fantasy; figured bass; Fuge; fugue; Generalbass; Improvisation; partimento; procedural knowledge; prozedurales Wissen

Improvisation im Musikstudium - das scheint auf den ersten Blick kein neues Thema zu sein. In der Kirchenmusik, im Jazz und beim klavierpraktischen Spiel wird improvisiert. Nicht zu vergessen das Spielen von Kadenzen und das mehr oder weniger geliebte Generalbassspiel in der Musiktheorie. Wer nun glaubt, das vorliegende Compendium Improvisation würde in einer dieser Traditionen stehen, der irrt. Der Herausgeber Markus Schwenkreis und seine Autor*innen verfolgen ein ganz und gar anderes Ziel. Nicht die Ergänzung der oben genannten Studien durch ein wenig Improvisation ist beabsichtigt. Vielmehr soll durch die Vermittlung einer besonderen improvisatorischen Sichtweise ein grundsätzlich anderer Zugang zur historischen Musikpraxis ermöglicht werden.

Wie im Untertitel angedeutet, basiert das Buch auf historischen Quellen des 17. und 18. Jahrhunderts. Im Mittelpunkt steht aber nicht primär das Zusammentragen von historischen Informationen, sondern die Aneignung praktischer Fähigkeiten. Mit der höchst lesenswerten Publikation wird ein tiefer Einblick in eine Musizierpraxis gegeben, welche denen eher fremd ist, die ihre »historischen Informationen « ausschließlich den musikwissenschaftlichen Quellen entnehmen.

Schwenkreis betont im Vorwort, dass ohne den regelmäßigen Austausch über methodische Fragen innerhalb der Forschungsgruppe Basel für Improvisation (FBI) die Entstehung des Compendiums nicht denkbar gewesen wäre. Und so treten neben Schwenkreis mit Nicola Crumer, Emmanuel Le Divellec und Sven Schwannberger drei weitere Mitglieder dieses FBI-Kollektivs als Autor*innen in Erscheinung. Aber auch die anderen neun Beitragenden stehen durch Studium oder Lehre in enger Verbindung zu der
Schola Cantorum Basiliensis (SCB) bzw. zur dort angesiedelten FBI: Florian Bassani, JörgAndreas Bötticher, Markus Jans, Johannes Menke, Nicoleta Paraschivescu, Annette Unternährer-Gfeller und Jean Claude Zehnder. Die Organisten Rudolf Lutz und Gaël Liardon setzten erste Impulse für einen Austausch von Unterrichterfahrungen, wobei ersterer sowie Schwenkreis mit jeweils sechs Beiträgen die Hauptautoren des großformatigen Compendiums sind.

Das Inhaltsverzeichnis des 408 Seiten umfassenden Werkes zeigt die Bündelung der 25 Beiträge zu fünf Kapiteln: 1. Generalbass, 2. Figuration und Variation, 3. Partimento, 4. Choral und 5. Präludium und Fuge - Die Kunst des Fantasierens.

Dem Vorwort des Herausgebers wird ein programmatisches Zitat vorangestellt, das Konfuzius zugeschrieben wird: »Erkläre mir und ich vergesse. Zeige mir und ich erinnere. Lass es mich tun und ich verstehe. «

Sehr zutreffend wird auf einen gewissen Anachronismus des derzeitigen Musiklebens hingewiesen: Auf der einen Seite bemühe man sich um eine historisch informierte Aufführungspraxis, auf der anderen Seite stünde immer noch die Interpretation der großen Meisterwerke in einer Art und Weise im Zentrum der Ausbildung, die für die Zeit vor 1800 bei weitem nicht angemessen sei. Wichtiger sei die Wiederbelebung der schriftlosen Musik, denn die schriftlich überlieferten Werke aus dieser Zeit müssten mit der »Brille des Improvisators« betrachtet werden (1).

Ebenfalls sehr zutreffend wird eingeräumt, einfachste Zusammenhänge ließen sich gegebenenfalls besser am Instrument als in einem schriftlich überlieferten Text demonstrieren. In der Tat verweigert sich die soral tradition`, der 
diese Improvisationspraxis entstammt, dem Medium Buch. ${ }^{1}$

Den grundlegenden Ansatz wird man gut nachvollziehen können. Spielarten der Improvisation prägten bis zum Ende des 19. Jahrhunderts die gesamte Praxis sogenannter klassischer Musik. Insofern könne - so die Feststellung von Nicola Cumer - der historisch informierte Zugang zu älterer Musik auf die Erforschung improvisatorischer Verfahren nicht verzichten. ${ }^{2}$ Diese Kunst werde »vor allem durch den direkten Kontakt zwischen Lehrer und Schüler am Instrument vermittelt« (4). Trotz dieser berechtigten Bedenken gegen das Medium Buch haben sich die Autor*innen erfreulicherweise zum Verfassen ihrer Texte durchgerungen.

Die meisten Quellen zur Improvisation, welche in diesem Buch eine breite Darstellung erfahren, liegen selbstverständlich sunterhalb des höchsten Gipfels der Musikı. Im Mittelpunkt steht zunächst eine grundlegende Entwicklung von handwerklichen Fähigkeiten. Dazu bietet das Lehrbuch unzählige und vielfältig angelegte praktische Übungen. Das Compendium verfolgt aber darüber hinaus auch das Ziel, künstlerische Absicht und Affektausdruck ebenfalls zu berücksichtigen (5).

In einem einführenden Kapitel führt Schwenkreis Quellen zu den sogenannten »Organistenproben« auf. Für einen Organisten galt es als eine "erbärmliche Sache», wenn man nicht in der Lage war, Musik zu improvisieren (9). In diesem Zusammenhang galt die Unterweisung im Literaturspiel in der ersten Hälfte des 18. Jahrhunderts niemals als Selbstzweck, sondern diente vor allem dazu, sich bei seinem eigenen kreativen Schaffen durch andere Modelle und Einfälle anregen oder korrigieren zu lassen $(12,14)$. Diese auch von Jakob Adlung (1758) bezeugte Methodik spielt für das vorliegende Compendium eine grundlegende Rolle.

1 Schwenkreis bestätigt an anderer Stelle, es sei unmöglich, den »in lebendigem Dialog erfolgenden Wissenstransfer einer oralen Tradition « - wie er in der Partimentotradition stattgefunden habe - zu verschriftlichen $(2012,309)$. Der vermeintliche Umweg über die Praxis sei der beste Weg, diese alte Kunst »aus ihrem Innersten heraus zu verstehen« (ebd., 310).

2 Vgl. Haselböck 1966.
Insgesamt wird das Konzept verfolgt, zunächst eine strenge Orientierung an konventionellen Generalbass-Methoden zu geben und dann im Verlaufe des Buches diese »Leitplanken « mehr und mehr zurückzunehmen um schließlich zur »Königsdisziplin« der Fugenimprovisation zu gelangen (15). Dabei sei das analytische Hören von großer Bedeutung, denn das Studium entsprechender Werke müsse keinesfalls immer nur über die Analyse des Notentextes erfolgen (17). Improvisation dürfe aber durchaus nicht als ein rationaler Vorgang missverstanden werden, sondern könne durchaus auch in einem rauschähnlichen Bewusstseinszustand ausgeführt werden und führe im Idealfall zu einem Stadium der Selbstvergessenheit, zu einem Flow (19).

\section{KAPITEL 1: GENERALBASS}

Schwenkreis beginnt den Hauptteil seines Kapitels mit einem starken Votum für die Bedeutung des Generalbasses. Ob dies allerdings bezogen auf Bachs vielfältiges Musikdenken ganz so zutrifft, wie vom hier zitierten zweitältesten Bachsohn behauptet (22), ist von namhaften Bachforschern bezweifelt worden. ${ }^{3}$

Die nachfolgende Systematik des ersten Kapitels zu den Schlüssen, Gängen und Sätzen entnimmt Schwenkreis der Anleitung zu der musikalischen Gelahrtheit von Jakob Adlung (29). Eine ähnliche Methodik weist der Autor für die neapolitanische Partimentotradition nach (31). Bekanntlich zeigten sich zwischen Komposition und Improvisation bis zur Mitte des 18. Jahrhunderts vielfältige Wechselwirkungen. Aus einer Grafik (32) geht hervor, dass aus den Kompositionen durch Vereinfachung und Entfigurierung abstrakte Satzmodelle extrahiert werden können, die dann auf dem Wege der Improvisation durch Transformation und Figuration wieder zu Musik werden. Der Kreislauf kann geschlossen werden, indem wiederum durch Verschriftlichung und Nachkorrektur Kompositionen entstehen. Im Mittelpunkt der musiktheoretischen Auseinandersetzung steht die praktische Erprobung und das

3 Schulze 1988, 469; Schulze spricht sogar von dem »Problem der gelegentlichen Unaufrichtigkeit des zweitältesten Bachsohnes « (ebd., 470), vgl. Felbick 2012, 161-164. 
Ausprobieren dieser Satzmodelle am Instrument, wobei die eigene »Hörvorstellung und die grifftechnische Realisation Hand in Hand gehen« (33). Die Übersicht zu den wichtigsten Satzmodellen des Generalbasszeitalters regt zu praxisorientierten Übungen an (34f.). Im Abschnitt zu den Schlüssen wird vielfach auf Georg Muffats Schrift Regulae Concentuum Partiturae (1699) Bezug genommen, denn Muffat äußert sich dort umfassend und systematisch zur Kadenzthematik (42-55). Der 13-taktige sogenannte »Dandrieu-Parcours« $(42,44)$, eine Kadenzfolge mit Zwischenstationen auf den wichtigsten Haupt- und Nebenstufen in Dur, dient als Übungsgrundlage für mannigfaltige Variationsübungen.

Insgesamt bietet das Buch schier unzählige Beispiele für satztechnische Konstellationen aus der Originalliteratur und zeigt vielfältige Wege auf, die große Zahl der zum Teil komplexen Tonbeziehungen zu kategorisieren, so beispielsweise die hervorragende Matrix zu den möglichen Klangfolgen, die aus einer Bassdiminution der scadenza doppiar resultieren können (62). Auf viele Möglichkeiten für den kreativen weiteren Umgang mit Satzmodellen wird hingewiesen (65).

Im Abschnitt zu den Gängen und Sequenzen heißt es, diese stünden den meisten Spielern zwar wie ein passiver Wortschatz einer Fremdsprache zur Verfügung. Wer aber eine barocke Musiksprache wirklich fließend beherrschen wolle, müsse sich die Grundmodelle als einen aktiven Wortschatz aneignen. Die entsprechenden Modelle müssten »begriffen « und in ihren zahlreichen Varianten in möglichst vielen Tonarten erarbeitet werden (67).

Besondere Aufmerksamkeit widmen die Autor*innen den zum Teil sehr kreativen Wortschöpfungen und Benennungen von bislang nicht bewusst erfahrenen Phänomenen. Beispielsweise leuchtet der Ausdruck "GabelgriffKanon« (94) denjenigen sofort ein, die diese zweistimmigen Modelle gespielt haben. Das immer wieder erkennbare Bemühen um die Schaffung neuer Kategorien zeugt von einer intensiven Beschäftigung mit der Thematik. "Strebeakkorde der Oktavregel « sind demzufolge nicht nur die auflösungsbedürftigen dominantischen Akkorde, sondern dazu zählt auch der Akkord mit der Dissonanz zwischen der Quinte und der Sexte auf der subdominan- tischen vierten Stufe (110). Eine solche besondere Kategorie ist sinnvoll, denn diese Strebeakkorde haben die Gemeinsamkeit, nicht sprungweise verlassen werden zu können. Die bei Schwenkreis vielfach erscheinende neue Begrifflichkeit deutet auf ein neues musikalisches Denken, welches sich von bisherigen theoretischen Erwägungen und Beschreibungen unterscheidet. Funktionstheoretische Begriffe, die von Puristen im Zusammenhang mit der Barockmusik gerne als anachronistisch gebrandmarkt werden, werden hier zielführend angewandt, z. B. wenn von zwischendominantischen Wendungen die Rede ist. Offensichtlich ist den Autor*innen das praktische Ergebnis des Musikdenkens wichtiger als eine terminologisch-historisch »exakte« Ausdrucksweise (116). Die Autor*innen ermutigen aber auch die Leserschaft, eigene ganz persönliche Namen zu suchen (70).

Sehr überzeugend ist die in diesem Zusammenhang angewandte Methodik, nach einer kurzen Übersicht den entsprechenden Begriff zu diversen Sequenzen zu erläutern und mit Literaturbeispielen anschaulich zu machen und dann die »Regula" in Form eines Notenbeispiels mit einem passenden Partimento aufzuführen (71). Ausführungen zur Oktavregel, zum Orgelpunkt und den Eröffnungsmodellen dürfen in einer solchen Darstellung nicht fehlen (103-131).

\section{KAPITEL 2: FIGURATION UND VARIATION}

Nach einem kurzen Beitrag, der sich zu Beginn des relativ knappen 2. Kapitels vornehmlich der Analyse von Klauseldiminutionen widmet (134-139) folgen zwei Workshops zu ostinaten Bässen. Zunächst steht der 'Lamentobass` bzw. die `Passacaglia im Mittelpunkt. Die Übungen zu den zehn kurzen Abschnitten sind auch von Anfängern gut umzusetzen. Besonders beeindruckend ist der Bezug zu einem Werk von Henry Purcell aus den Ten Sonatas in four parts (150). Der Komponist lässt in diesem fünftaktigen Ostinato die Kadenz an einigen Stellen wandern. Dies führt zu Kadenzabschlüssen an unterschiedlichen Stellen des Ostinatos und damit zu interessanten Asymmetrien. Durch die dargestellte Reduzierung dieses kompositorischen Prinzips auf einen einfachen Gerüstsatz ist dieser kompositorische »Trick" sehr gut 
nachvollziehbar. Es ist durchaus positiv zu werten, dass Schwenkreis hier auf die Detailanalyse verzichtet, denn so kann das für diese Thematik Wesentliche herausgestellt werden. Außerdem gibt Schwenkreis in seinem Text Ausführungen zum dreifachen Kontrapunkt (152f.). ${ }^{4}$

Auf der Basis einer Idee von Rudolf Lutz folgt nun sein zweiter Workshop, dieses Mal zum `Folia«-Bass. Durch die Einführung von verschiedenen gut nachvollziehbaren Begriffen wie beispielsweise "5-8-Schwebestimme« gelingt es dem Autor, den Improvisationsschüler*innen konkrete Spielanweisungen an die Hand zu geben (155). Die Lernenden müssen das Grundmodell auswendig beherrschen und mit der von ihren Lehrer*innen verwendeten Spezialterminologie vertraut sein. So lässt sich erahnen, wie ein solches Gespräch im konkreten Improvisationsunterricht stattfinden könnte. Dazu wäre ein ins Internet eingestelltes exemplarisches Video eines solchen Unterrichtsdialogs als multimediale Ergänzung des Buches hilfreich gewesen.

Der Autor gibt die Empfehlung, sich beim Üben mit geschlossenen Augen voll und ganz auf das Hören zu konzentrieren (155). Dieser primär auditive Ansatz entspricht der Denkweise der oralen Kultur, die immer wieder als Richtschnur eines derartigen Unterrichtes betont wird. Bestimmte Bewegungsabläufe beim Improvisationsvorgang werden als automatisiert bezeichnet, verbunden mit dem kreativ formulierten Hinweis, man sollte in solchen Momenten auf "Autopilot" umschalten (158). Die Anregung, eine ‘Folias beispielsweise auch im Duo mit einem $/ \mathrm{r}$ weiteren Spieler*in eines Melodieinstrumentes zu improvisieren, eröffnet die Möglichkeit, den Improvisationsunterricht nicht nur auf Tasteninstrumente zu beschränken (160), wie dies den Inhalt des Buches größtenteils bestimmt.

Die musiktheoretischen Formulierungen des Compendiums sind meist auf Einfachheit und konkrete Umsetzbarkeit hin angelegt. Das entspricht durchaus dem Musikdenken improvisierender Musiker*innen, denn diese können möglicherweise mit einer praxisbetonten Formulierung wie "Vorhaltsbildungen in den Gymelstimmen« (160, 161, Bsp. 8-B) mehr anfangen als mit der skorrekten Beschreibung sämtlicher tonaler Verhältnisse.

\section{KAPITEL 3: PARTIMENTO}

Nach den Erläuterungen zur Geschichte und Lehrmethode der neapolitanischen >Partimentopraxis» im nachfolgenden dritten Kapitel folgt ein praxisorientierter Lehrgang von Rudolf Lutz. Selbstverständlich sei eine Erfahrung im Generalbassspiel unabdingbare Voraussetzung für einen solchen Unterricht. Die Fähigkeit, beim bloßen Anblick einer unbezifferten Basslinie die möglichen Oberstimmen innerlich zu hören, gelte als didaktisches Ziel. Diese Fähigkeiten wurden in den Konservatorien in Neapel im Laufe eines etwa zehnjährigen Studiums erworben $(166,167)$. Weiterhin sei eine stilgemäße Ausführung der >Partimentiء ohne eine entsprechende Kenntnis der italienischen Tastenliteratur kaum vorstellbar (172). Die Bewältigung dieses recht umfangreichen Lernpensums führe zu einer hervorragenden Schulung des kontrapunktischen Denkens und dem gründlichen Verständnis musikalischer Zusammenhänge. Lutz hat sich zweifellos intensiv mit diesem Nukleus der barocken Musik beschäftigt. Deshalb ist ihm auf der Basis dieser improvisatorischen Kompetenz ein tiefer Einblick in diese Epoche möglich. Diese an der Improvisation orientierte »Ausbildung, die einen weiten Erfahrungshorizont für Lernende und Ausführende erschließt«, empfiehlt er allen Musiker*innen (178). Das bedeutet im Klartext eine Verschiebung der in erster Linie auf technische Höchstleistung angelegten heutigen Standardausbildung im Curriculum eines Musikstudiums dahingehend, dass das Fach Improvisation ganzheitlich und intensiv, nach der im Compendium beschriebenen Weise gelehrt und gelernt wird.

Bei der Anleitung zur Improvisation einer Suite kommt den Menuetten nicht die zentrale Rolle zu, wie sie beispielsweise durch Joseph Riepel bezeugt ist. Der mit vielen Musikbeispielen erläuterte Text bezieht sich im Wesentlichen auf die Lehrmethode, die Friedrich Erhardt Niedt im zweiten Teil seiner Musicalischen Handleitung darlegt. Aus ein und demselben Partimento entstünden durch kleine Anpassungen verschiedene Suitensätze (183). Die Autorin Annette Unternährer-Gfeller über- 
nimmt diese Methode zwar grundsätzlich von Niedt, verfasste aber ein eigens für diesen Artikel komponiertes Partimento. Die zudem beigefügten Gerüstsätze sind beim Studium am Instrument sehr hilfreich. Selbstverständlich steht vor der konkreten Improvisation die intensive Beschäftigung mit der Struktur des vorgegebenen Basses und den satztechnischen Gegebenheiten. Dieser Harmonieablauf soll zuvor in allen möglichen Konstellationen sdurchdekliniert werden (186). Als Erweiterung der Methode wird ein Rhythmus-Partimento vorgestellt, bei dem über dem Bass der Rhythmus einer möglichen Oberstimme notiert ist. Die Methode der Orientierung an einem vorgegebenen Rhythmus hätte beispielsweise bei der Menuettimprovisation durchaus noch weiter ausgeführt werden können. Dabei wäre auch die Arbeit mit einstimmigen Menuettentwürfen sinnvoll gewesen, zumal diese in den musiktheoretischen Quellen viel häufiger zu finden sind als ein sofortiger Einstieg in die Zweistimmigkeit.

Ein musikwissenschaftlich angelegter Beitrag von Florian Bassani zu den Partimentofugen in deutschen Quellen bildet den Abschluss des dritten Kapitels (199-209).

\section{KAPITEL 4: CHORAL}

Beim vierten Kapitel werden vor allem die verschiedenen Aspekte des protestantischen Chorals behandelt, zu dem Rudolf Lutz zunächst eine kurze Einführung gibt. Die Kunst der Choralharmonisation könne "zum Brennpunkt der barocken Kompositionslehre, zu einer Art Wörterbuch der Affektdarstellung werden. « Zweifellos können die tonalen Verhältnisse in den Chorälen in der Renaissance und Barockzeit »zum Urbild musikalischer Prozesse« werden (213f.).

Die folgenden fünf Beiträge zeigen unterschiedliche Aspekte dieser Choralarbeit. Emmanuel le Divellec schreibt, man müsse beim improvisatorischen Prozess alle Möglichkeiten der Stimmführung nicht nur kennen, sondern man müsse sie auch können, das heißt spielpraktisch beherrschen (217). Anhand der ersten beiden Choralzeilen von »Herr Christ, der einig Gotts Sohn « zeigt er 27 verschiedene homophone und polyphone satztechnische Konstellationen auf. Primär soll diese Arbeit improvi- sierend am Instrument geschehen, wobei bei bestimmten Aufgabenstellungen eine Ausführung nur an der Orgel möglich ist. Die beim Spiel erarbeiteten wenigen Takte sollten nachfolgend aus dem Gedächtnis aufgeschrieben werden, um das Ergebnis in aller Ruhe kontrollieren zu können. Dass diese Arbeit sehr viele Stunden beansprucht und nicht beiläufig neben dem vermeintlich wichtigeren Literaturspiel erledigt werden kann, ist offenkundig. Der Autor mahnt zu großer Disziplin und räumt gerne ein, das konsequente Durcharbeiten der vorgegebenen Muster sei durchaus eine große Herausforderung, quasi eine "ermüdende Knochenarbeit« (217). Man müsse sie als notwendige »Deklinationsübungen « verstehen. Dieser der Grammatik entlehnte Begriff scheint für diese Aufgabenstellungen sehr zutreffend, denn aus einer Grundform entstehen hier und dort verschiedene weitere Gestalten, die jeweils einer gewissen Gesetzmäßigkeit folgen.

Mit den Zeilenzwischenspielen in einer Choralbegleitung befasst sich ein relativ umfassender Beitrag von Jörg-Andreas Bötticher. Diese Improvisationspraxis lässt sich durch einige Quellen nachweisen, ist aber vielfach auch umstritten. Kritische Stimmen äußerten im 18. Jahrhundert beispielsweise, »dass Organisten in diesem Bereich mitunter offenbar ein Tummelfeld für ihre kreativen improvisatorischen `Ergüsse sahen. « Dies könne in der singenden Gemeinde zur Verwirrung führen (229). Diese Thematik wird nur spezielle Interessenten ansprechen. Man mag den folgenden Text von Rudolf Lutz, der ebenfalls unterschiedliche Möglichkeiten anhand von zwei Choralzeilen demonstriert, als Fortsetzung der ,Deklinationsübungen betrachten. Auch diese Arbeit basiert auf dem Umgang mit verschiedenen Choralbausteinen. Diese werden in einer tabellarischen Übersicht (247) und in einer Ergänzung von Markus Jans (250) sehr inhaltsreich dargestellt. Sie seien auswendig zu spielen und in die gebräuchlichsten Tonarten zu transponieren.

Im folgenden Beitrag von Schwenkreis zu den Variationstechniken der mitteldeutschen Aria variata und Choralpartita demonstriert der Autor seine hervorragende Unterrichtsmethode und Didaktik. Er setzt sich mit dem Vorwurf 
Willi Apels ${ }^{5}$ auseinander, die Variationen des späten 17. Jahrhunderts seien von einer gewissen Formelhaftigkeit. Gerade diese einfachen Variationsmuster hätten als handwerklich gediegene Vorbilder aber zu den »Pflichtübungen des täglichen Improvisationsunterrichtes` gehört (251). Deshalb sei der Mangel an kompositorischer Fantasie für die pädagogische Zielsetzung völlig ohne Belang.

Bei der Aria variata wird nicht nur der Bass, sondern auch die Oberstimme mehr oder weniger vorgegeben (251). So bildet sich ein "zweistimmiges harmonisches Gerüst, welches in den einzelnen Veränderungen figuriert und umspielt wird«. Schwenkreis führt dazu 32 Beispiele an und empfiehlt deren Studium. Ausgangspunkt der nachfolgenden Übungen ist ein schriftlich vorliegender dreistimmiger Gerüstsatz zu einem Choral in einer , [1-2]Disposition (Melodie rechts, Bass und Mittelstimme links). Diese systematisch verwendete Ausdrucksweise für die Verteilung der zu spielenden Töne auf die Hände (bzw. das Pedal) ist für die Praxis der Improvisation recht hilfreich (vgl. diesbezügliche Ausführungen auf S. 2). Ebenso hilfreich sind die Kategorisierungen von in der Regel viertönigen Sechzehntelmotiven inklusive einer zum Teil unkonventionellen, aber unmittelbar einleuchtenden Begrifflichkeit, zumal das »Frère-Jacques-Motiv« auch als Umkehrung, Krebs oder Krebsumkehrung gedacht werden kann und in dieser Stilistik häufig auftritt. Der Autor empfiehlt konkrete Übungen zu seinen systematischen Motivkategorien wie z. B. eine »Tetrachord-Deklination« zu einem vorher gegebenen Gerüstsatz (259).

Der auf zwei Manualen und Pedal auszuführende kolorierte Orgelchoral gehört zu den Standardaufgaben einer traditionellen Organistenausbildung. Die diesbezüglichen Ausführungen von Le Divellec basieren auf Choralvorspielen von Heinrich Scheidemann (15961663) und Dietrich Buxtehude (1637-1707). Die Werke dieser Komponisten seien ideale Vorlagen, die sich gegenseitig ergänzten, die allerdings auch eine stilistische Schnittmenge aufwiesen. Bei Buxtehudes Verzierungskunst werden wesentliche Manieren zum Ausdruck eines Affektes verwendet. Somit ist dessen rhythmische Vielfalt noch größer als die Scheidemanns (271).

Die komprimiert dargestellte Thematik wird übersichtlich gegliedert und die Ausführungen sind durch zahlreiche knappe Zitate aus der Originalliteratur leicht nachvollziehbar. Besonders beeindruckend ist die Unterscheidung zwischen den "kopfgesteuerten " Figuren und den eher intuitiv gefundenen Figuren. Erstere wirkten nicht nur stilfremd, sondern auch künstlich. Durch intensives und konsequentes Üben kämen diese passenden Figuren dann aber automatisch »in die Finger «. Diese hier mitgeteilte improvisatorische Erfahrung kann der Rezensent bestätigten.

Zusätzlich zur eigentlichen Kolorierungsthematik werden Hinweise zu fugierten Stimmeinsätzen gegeben, die am Anfang einer derartigen Choralbearbeitung oder als Zwischenspiele erscheinen können (273).

\section{KAPITEL 5: PRÄLUDIUM UND FUGE - DIE KUNST DES FANTASIERENS}

Das letzte Kapitel behandelt Präludium und Fuge als den höchsten Grad der Improvisationskunst. Der Autor Lutz berichtet, Präludien seien als ein Sammlerkabinett der verschiedensten Bautechniken zu verstehen, die in ihren Potenzialen erkundet werden sollten. Diese unterschiedlichsten Ideen könnten für eigene musikalische Schaffensprozesse nutzbar gemacht werden. Mit diesem Ansatz demonstriert der Autor seine »historische Informiertheit«, denn dem zeitgenössischen Opus-Begriff war die Plagiat-Idee noch äußerst fremd. Lutz räumt ein, in der Praxis des Extemporierens werde in der Regel mit relativ überschaubaren Modellen gearbeitet. Mit derartigen um 1700 bei Johann Christoph Bach (1673-1727) nachweisbaren Kompositionen, einer Sammlung einfacher Präludien, beschäftigt sich nachfolgend Jean-Claude Zehnder. Sehr stiltypisch ist in den vielfach anonym überlieferten Werken die Satztechnik durezze e ligature, in der es durch Überbindungen von Akkorden zur nachfolgenden schweren Zählzeit und gleichzeitig sich ändernder Bassstimme zu einer Vielzahl von mehrstimmigen Vorhaltsdissonanzen kommt (297).

Zwar liefern Markus Jans und Rudolf Lutz zum Thema Modulation zahlreiche Analysen, 
allerdings steht die konkrete Anwendung immer im Mittelpunkt. Der damit verbundene auditive Vorgang wird in interessanten Details lebhaft auf erfrischende und fantasievolle Weise erläutert, denn man spüre »im Korridor vielleicht etwas Zugluft vermischt mit Düften aus der Küche, betritt den neuen Raum, setzt sich an den Esstisch... (305). Die gelegentlich anzutreffende terminologische Verwirrung zwischen den Begriffen Ausweichung und Modulation vermeidet Jans durch seine Definition der Modulation als das auditiv wahrgenommene »Ablösen eines vorhandenen durch ein neues tonales Zentrum « (305). Nach den notwendigen theoretischen Erörterungen folgt ein praktischer Teil zur Modulation von Lutz (309). Hier finden sich neben Analysen wichtige Hinweise zum musiktheoretischen Denken von improvisierenden Musiker*innen.

Der folgende Abschnitt von Nicola Cumer wird mit dem Thema "Sonar di fantasia im 17. Jahrhundert« wieder sehr konkret, zumal die reiche Tradition des contrappunto alla mente weiterhin die Basis der hier präsentierten Musikpädagogik bleibt. Die in Girolamo Dirutas /I Transilvano ausführlich beschriebene Technik des ıIntavolierens« stellt eine wichtige Voraussetzung für die Variationskunst dar und bildet hier den Ausgangspunkt des ersten Teils der Darstellung (319). Diese Technik wird im 16. und 17. Jahrhundert als Grundvoraussetzung betrachtet, um im polyphonen Stil der Zeit extemporieren zu können (320). Nach der ausführlich vorgestellten Praxis der intavolatura diminuita folgen entsprechende Übungen. Die Auffassung, man könne so die italienische Unterrichtspraxis zu den Clavier-Variationen des frühen 17. Jahrhunderts rekonstruieren, wird nachvollziehbar am Beispiel erläutert, wobei zunächst von Modellen ausgegangen wird, die nur aus wenigen Akkorden bestehen (326). Folgerichtig führt der Unterricht zur Improvisation polyphoner Diminutionen und den passaggi (329ff.). Sowohl diese Anweisungen als auch manch andere Aufgabenstellungen des Buches erinnern ein wenig an ähnliche Aufgabenstellungen des Jazz. Dieser Eindruck verstärkt sich beim Abschnitt Sonator da Balli, denn gleichbleibende ,Changes» werden hier und dort in verschiedenen Taktarten und 'Sty- les` zu sinnvollen musikalischen Einheiten gefügt (335ff.). ${ }^{6}$

Der zweite Teil widmet sich den Grundlagen der imitatorischen Improvisation, wobei zu der kontrapunktischen Gattung der >Versetten jeweils kleine Bausteine hinzugefügt werden und so komplexere Formen der 'Canzonar entstehen (341). Man möge aber zu Beginn nicht die Canzonen Frescobaldis als Muster betrachten, denn diese seien zwar als der Gipfelpunkt der Gattung anzusehen, aber zunächst zu komplex (342).

Dieser Abschnitt stellt eine gute Vorübung für den nachfolgenden Beitrag "Übungsfelder zur Fugenexposition « von Rudolf Lutz dar (347). Die elf Unterabschnitte ähneln zwar oft einem Lehrbuch zu dieser "Königsdisziplin«, aber der Autor gibt immer wieder auch wichtige Hinweise, die man in derartigen Unterrichtswerken nur vereinzelt findet. Er verweist beispielsweise auf die Bedeutung der Hörerfahrungen, welche auch intuitiv durch einen spielerisch-improvisierenden Umgang mit dem Thema gewonnen werden könnten. So kristallisierten sich »beim Fantasieren die harmonischkontrapunktischen und rhythmisch-metrischen Problemfelder des Themas heraus" (350). Neben der Bedeutung des Memorierens wird beispielsweise bei der Improvisation einer dreistimmigen Fuge auf den vorher festzulegenden Plan verwiesen, wobei von den sechs möglichen Einsatzdispositiven einige besonders häufig benutzt würden. Neben Analysen seien - unter Verweis auf eine Empfehlung Matthesons - auch schriftlich ausgearbeitete Fugen ein Mittel, die musikalische Denkweise der Fugenimprovisation zu vervollkommnen (354).

Die Fugen von Johann Pachelbel wählte Gaël Liardon in seinem Beitrag als Improvisationsmodelle. Zweifellos eine gute Wahl, denn diese oftmals kleinen Werke eignen sich bestens für pädagogische Zwecke, viel besser als dies die traditionelle Unterrichtstradition der fugue $d$ 'école vermittelt. Diese an Bach'schen Fugen angelehnten Modelle seien oft zu komplex und für durchschnittlich begabte Improvisationsschüler*innen auch nach langem Studium immer noch unerreichbar (355).

6 Sikora 2003, vgl. die Klangbeispiele auf den Sikoras Buch beiliegenden CDs. 
Obwohl Pachelbel auch geringstimmigere Fugen komponierte, stehen hier die über hundert vierstimmigen Kompositionen im Fokus. Der Autor wählt eine Terminologie, die sehr ungewöhnlich - aber hinsichtlich der Aufgabenstellung durchaus pragmatisch erscheint. Mit wenigen Zeichen kann so ein FugenSchema nach Pachelbel grob skizziert werden, sodass die hier vorgestellte spezielle analytische Kurzschrift in Kombination mit der einprägsamen Darstellung der üblichen Einsatzdispositive hervorragend als Vorlage für Fugenimprovisationen dienen kann (359). Hier könnte man fragen, ob nicht noch der Hinweis auf noch einfachere Fugenthemen für den Beginn hilfreich wäre, wie die an anderer Stelle vorgestellten Tonfolgen des »Frère-Jacques-Motivs" (255). Dieses Motiv kann in vier isometrischen Werten sowohl in Dur als auch in Moll als Umkehrung, Krebs oder Krebsumkehrung als ein einfaches Thema verwendet werden. Die Rezeption eines solchen sehr schlichten Fugenthemas - in diesem Falle nach dem im Fux'schen Lehrbuch enthaltenen Muster - wird noch in den Aufzeichnungen Beethovens im Zusammenhang mit seinem Fugenstudium bezeugt. ${ }^{7}$ Bereits Georg Andreas Sorge hatte 1745 im Zusammenhang mit seinen Ausführungen zum "compositor extemporaneus" darauf hingewiesen, dass der heute oft missverstandene Fux im zeitgenössischen Improvisationsunterricht benutzt wurde, denn man lerne "aus Herrn Matthesons Kern melodischer Wissenschafften und Fuxens Gradibus ad Parnassum eine gute Fuge machen, und ein gewisses Thema oder Subjectum extempore auszuführen. $«^{8}$

Übergeordnete Aspekte »Rhetorik - Fantasieren als musikalischer Redekunst « werden abschließend von Schwenkreis inklusive eines

7 Beethoven hat das einfache Thema $d$-f-e- $d$ bei seinen Fugenstudien bzw. für seine Unterrichtstätigkeit aus dem Fux'schen Gradus ad Parnassum rezipiert, wobei der Komponist nachweislich die deutsche Gradus-Übersetzung Mizlers besaß; vgl. Autograph Beethovens im Beethoven-Haus Bonn, Sammlung H. C. Bodmer, HCB Mh 45.

8 Sorge 1745, Teil III, 425. Sorge gibt diese Empfehlung im 30. Kapitel, welches Hinweise enthält, wie man ein guter »Compositor extemporaneus « werden könne.
Intermezzos von Lutz behandelt. Die fünf Teile, die nach Quintilian in der Rhetoriklehre behandelt werden, gliedern diesen letzten Beitrag. Die Inventio beinhaltet das für die Rede benötigte Material. Schwenkreis folgt den »Erfindungs-Qvellen", die Mattheson unter Berufung auf Christoph Weißenborn aufführt. ${ }^{9}$ Adlung rät, ein Büchlein mit guten sThematas anzulegen ${ }^{10}$ und Scheibe und Kittel geben weitere Anregungen hierzu (366). Die klassischen Teile einer Rede werden in der Dispositio behandelt, hier ergänzt durch Anregungen aus historischen Quellen. Neben allen technischen Voraussetzungen sei aber auch der Affektausdruck (Elocutio) wichtig, der in den musikalischen und rhetorischen Figuren zum Ausdruck kommt und zu dem zahlreiche Quellentexte aufgeführt werden.

Der vierte Teil der Rhetorik betrifft die Improvisation in besonderem Maße, denn eine geschulte Memoria beinhalte u. a. die Fähigkeit, mit dem »in den Fingern " gespeicherten Wissen in Echtzeit »denken« zu können. Es müsse zwar auch auf ein memoriertes Material zurückgegriffen werden, welches dem deklarierten Wissen zugeordnet ist, in viel höherem Maße basiere die Memoria hier aber auf intuitivem Wissen (prozeduralem Wissen) und werde - vergleichbar dem Beherrschen einer Sprache - durch sehr lange praktische Erfahrung im Umgang mit den Grundmustern erlernt. Zu dieser Thematik bietet der Beitrag wertvolle Hinweise, die auch Aspekte der Kognitionspsychologie und Erfahrungsberichte aus dem Jazz beinhalten (380f.). Der letzte Teil der Rhetoriklehre behandelt die Pronuntiatio oder Actio, die konkrete Bühnenpräsentation des Künstlers.

In der ausführlichen Bibliographie werden die wichtigsten Quellen und die Forschungsliteratur vorgestellt und das angefügte Glossar dient als Hilfe für die terminologische Orientierung.

Mit dem Compendium Improvisation wurde zweifellos ein wichtiges Standardwerk zur Musik des 17. und 18. Jahrhunderts vorgelegt. Nach der Lektüre und der Ausführung der Übungen wird nachvollziehbar, warum ein tiefes Verständnis dieser Musik und der damaligen Musizierpraxis vor allem durch aktiven

9 Mattheson 1739, 123, § 21.

10 Adlung 1758, 751. 
Nachvollzug mit den Mitteln der Improvisation erreicht werden kann. Demzufolge deutet eine derartig starke Schwerpunktsetzung im Curriculum nicht auf eine Neuordnung eines Nebenfachs, sondern verweist auf eine grundlegende Reform des gesamten Studienplans, welcher in vieler Hinsicht an ein Jazzstudium erinnert. Mit dem Compendium Improvisation wurde zweifellos eine Pionierarbeit geleistet, die tiefer gehende Annäherungen an ältere Musik ermöglicht als sie bislang üblich waren. ${ }^{11}$

Selbst ein derart umfangreiches Compendium kann es nicht leisten, alle Aspekte der Improvisationskunst anzudeuten. ${ }^{12}$ Auch Georg E. Lewis und Benjamin Piekut gaben 2016 ein zweibändiges, 1163 Seiten umfassendes Handbook zur Improvisation heraus. Hier wurde beispielsweise das ästhetische Verhältnis der

11 Zwar hat Doll (1989) in großer Fülle Quellen aus achtzig Improvisationsschulen für Tasteninstrumente aus der Zeit von 1600 bis 1900 zusammengestellt und Konrad (1991) legte ein Lehrwerk mit ähnlichem Titel vor, aber ersteres ist in pädagogischer Hinsicht unbefriedigend und letzteres erfüllt in gewisser Hinsicht diesen Maßstab, überzeugt aber in musikwissenschaftlicher Hinsicht nicht und umkreist - anderes als das empfehlenswerte Buch Sikora (2003) zu viele Themen, wobei der Jazz dabei eigenartigerweise völlig ausgespart wird.

12 Beispielsweise machte die grobe Verunglimpfung der Improvisationskunst als eine »Missgeburt" in Leipzig in unmittelbarem Umfeld Bachs die Runde. Sie erfolgte durch keinen Geringeren als Johann Christoph Gottsched (1733, 244) und später dann durch dessen Schüler Mizler $(1740,97)$. Diese Quellen gelten als erste wichtige Zeugnisse für die allmählich aufkommende Gegnerschaft der Freien Fantasie. Diese von der Philosophie Wolffs geprägten Kritiker legten - ähnlich wie heute die GEMA Wert auf die visuelle "Nachvollziehbarkeit" eines akustischen Werkes. Ein nicht schriftlich verfasstes, aus dem Stegreif entstandenes Musikstück könne - so die nicht nachvollziehbare Logik - »nicht viel werth « sein (vgl. Felbick 2012, 87f.).
Improvisation zum Zeitbewusstsein (»TimeConsciousness «) erörtert. Dieses Handbook sei als Ergänzung zum Compendium empfohlen, denn bei der Darstellung des Phänomens der Improvisation sind die unterschiedlichsten Ansätze möglich. ${ }^{13}$

\section{Lutz Felbick}

13 In der angloamerikanischen Literatur wird die historische Improvisation 1500 bis 1750 weiterhin in der von Massimiliano (2017) herausgegebenen Schrift erörtert (vgl. Rabinovitch 2017). 


\section{Literatur}

Adlung, Jacob (1758), Anleitung zur musikalischen Gelahrtheit, Erfurt: J. D. Jungnicol.

Apel, Willi (1967), Geschichte der Orgel- und Klaviermusik bis 1700, Kassel: Bärenreiter.

Doll, Egidius (1989), Anleitung zur Improvisation: ein Lese- und Lernbuch, Regensburg: Bosse.

Felbick, Lutz (2012), Lorenz Christoph Mizler de Kolof - Schüler Bachs und pythagoreischer "Apostel der Wolffischen Philosophie" (= Schriftenreihe der Hochschule für Musik und Theater 'Felix Mendelssohn Bartholdy Leipzig, Schriften, Bd. 5), Hildesheim: Olms.

Froebe, Folker (2010), „Vom Tonsatz zum Partimento«, ZGMTH 7/2, 215-231. https://doi.org/ 10.31751/512 (27.6.2019)

Gottsched, Johann Christoph (1733), Erste Gründe der gesamten Weltweisheit, Bd. 1, Leipzig: Breitkopf.

Haselböck, Hans (1966), „Improvisation Thoughts about its History and Teaching", The Diapason 57, 46-47.

Konrad, Rudolf (1991), Kompendium der Klavierimprovisation, Wien: Universal Edition.

Lewis, Georg E. / Benjamin Piekut (Hg.) (2016), The Oxford Handbook of Critical Improvisation Studies, Oxford: Oxford University Press.

Massimiliano, Guido (Hg.) (2017), Studies in Historical Improvisation from Cantare super Librum to Partimenti, London: Ashgate.

Mattheson, Johann (1739), Der Vollkommene Capellmeister, Hamburg: Christian Herold.

Mizler, Lorenz Christoph (1740), Musikalischer Staarstecher, Leipzig: Selbstverlag.

Rabinovitch, Gilad (2017), „Review of Massimiliano Guido, ed., Studies in Historical
Improvisation from Cantare super Librum to Partimenti (Ashgate, 2017) ", music theory online 23/4. http://mtosmt.org/issues/mto. 17.23.4/mto.17.23.4.rabinovitch.html (17.6.2019)

Schulze, Hans-Joachim (1988), „Zur Herausbildung einer >Bachlegendes im 18. Jahrhundert", in: Bericht über die Wissenschaftliche Konferenz zum 5. Internationalen Bachfest der DDR [...] Leipzig 1985, hg. von Winfried Hoffmann, Leipzig: Deutscher Verlag für Musik.

Schwenkreis, Markus (2008), » Vom Fantasieren : Methodik der stilgebundenen Improvisation nach Jacob Adlung", Dutch Journal of Music Theory 13/1, 98-108.

Schwenkreis, Markus (2012), „Giorgio Sanguinetti, The Art of Partimento. History, Theory, and Practice, New York: Oxford University Press 2012«, ZGMTH 9/2, 307-312. https:// doi.org/10.31751/683 (27.6.2019)

Sikora, Frank (2003), Neue Jazz-Harmonielehre Verstehen-Hören-Spielen, Von der Theorie zur Improvisation, Mainz: Schott.

Sorge, Georg Andreas (1745), Vorgemach der musicalischen Composition oder: Ausführliche, ordentliche und vor heutige Praxin hinlängliche Anweisung zum General-Baß, durch welche ein Studiosus musices zu einer gründlichen Erkänntnis aller in der Composition und Clavier vorkommenden con- und dissonirenden Grundsätze [...] kommen, folglich nicht nur ein gutes Clavier als ein Compositor extemporaneus spielen lernen, sondern auch in der Composition selbst wichtige [...] Profectus machen kann, Lobenstein: Selbstverlag. 
Felbick, Lutz (2019): Markus Schwenkreis (Hg.), Compendium Improvisation - Fantasieren nach historischen Quellen des 17. und 18. Jahrhunderts (= Scripta, Bd. 5), Basel. Schwabe 2018. ZGMTH 16/1, 125-135.

https://doi.org/10.31751/999

(C) 2019 Lutz Felbick (Lutz@Felbick.de)

Robert Schumann Hochschule Düsseldorf

Dieser Text erscheint im Open Access und ist lizenziert unter einer Creative Commons Namensnennung 4.0 International Lizenz.

This is an open access article licensed under a

(c) (1)

Creative Commons Attribution 4.0 International License.

eingereicht / submitted: 31/01/2019

angenommen / accepted: 23/02/2019

veröffentlicht / first published: 30/06/2019

zuletzt geändert / last updated: 30/06/2019 